\title{
Explanation, geometry, and conspiracy in relativity theory
}

\author{
James Read*
}

\begin{abstract}
I discuss the debate between dynamical versus geometrical approaches to spacetime theories, in the context of both special and general relativity, arguing that (a) the debate takes a substantially different form in the two cases; (b) different versions of the geometrical approach - only some of which are viable - should be distinguished; (c) in general relativity, there is no difference between the most viable version of the geometrical approach and the dynamical approach. In addition, I demonstrate that what have previously been dubbed two 'miracles' of general relativity admit of no resolution from within general relativity, on either the dynamical or 'qualified' geometrical approaches, modulo some possible hints that the second 'miracle' may be resolved by appeal to recent results regarding the 'geodesic principle' in GR.
\end{abstract}

*james.read@philosophy.ox.ac.uk 


\section{Contents}

1 Introduction 3

\begin{tabular}{|lll}
2 & Background & 5
\end{tabular}

2.1 Spacetime theories . . . . . . . . . . . . . . . 5

2.2 Symmetries . . . . . . . . . . . . . . . . . . . . 6

2.3 Special and general relativity $\ldots \ldots \ldots \ldots$. . . . . . 7

2.3 .1 Special relativity $\ldots \ldots \ldots \ldots$. . . . . . . . . 7

2.3 .2 General relativity . . . . . . . . . . . . . 8

$\begin{array}{lll}3 & \text { The dynamical/geometrical debate } & 11\end{array}$

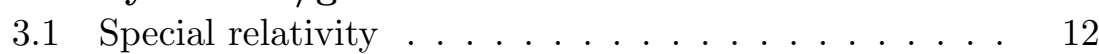

$3.1 .1 \quad$ The geometrical approach . . . . . . . . . . . . 12

3.1 .2 The dynamical approach . . . . . . . . . . . . . 13

3.2 General relativity . . . . . . . . . . . . . . . . . 13

3.2 .1 The geometrical approach . . . . . . . . . . . . . . 14

3.2 .2 The dynamical approach. . . . . . . . . . . . 14

$\begin{array}{lll}4 & \text { Qualified and unqualified explanations } & 15\end{array}$

$\begin{array}{lll}5 & \text { The geometrical approach } & 16\end{array}$

5.1 Two geometrical approaches . . . . . . . . . . . . . . 16

$5.1 .1 \quad$ Special relativity . . . . . . . . . . . . . . 17

$5.1 .2 \quad$ General relativity . . . . . . . . . . . . . . . 19

5.2 The qualified geometrical approach . . . . . . . . . 20

$5.2 .1 \quad$ Univocal explanation . . . . . . . . . . . . . . . . . 20

$5.2 .2 \quad$ Partial explanation . . . . . . . . . . . . . . . 21

$\begin{array}{lll}5.2 .3 & \text { Confident and cautious qualified approaches . . } & 25\end{array}$

5.3 Two miracles, reprise . . . . . . . . . . 26

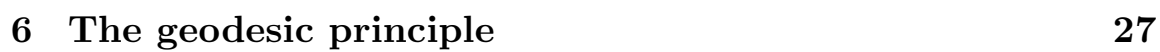

\begin{tabular}{lll}
\hline 7 & Conclusions & 34
\end{tabular} 


\section{Introduction}

It is roughly a decade since the groundbreaking work of Brown and Pooley [6, 7, 8, brought into the mainstream philosophy of physics literature the debate between dynamical versus geometrical approaches to spacetime theories. At the most general level, this debate centres upon the following question: whence the chronogeometric significance of the metric field? That is, why is the metric field (in theories such as special and general relativity) surveyed by rods and clocks built from matter fields? While advocates of the geometrical approach maintain that the metric field (in some sense) explains or constrains the form of the dynamical laws for matter fields, such that those fields behave such as to survey the metric field, advocates of the dynamical approach, by contrast, claim that an account of the chronogeometric significance of the metric field may begin from considerations regarding only the dynamical laws governing matter fields themselves.

Of course, this is vague; in $\$ 3$ of this paper, I sharpen significantly the above presentation of the debate. Nevertheless, even at this early stage, a number of genuine and substantial questions arise. Some of those which concern me in this paper are the following:

1. Does the dynamical/geometrical debate take the same form in the context of theories with fixed metric structure (such as special relativity (SR)) as it does in theories with dynamical metric structure (such as general relativity (GR))?

2. What notion of explanation is at play in this debate? Does answering this question reveal multiple different senses in which the dynamical/geometrical approaches may be understood?

3. Are the dynamical and geometrical approaches truly distinct from one another at all?

4. How does the dynamical approach relate to e.g. the 'spacetime functionalism' of Knox [32, 30, 31, or recent discussion on these matters by Weatherall? [69, $\S 6]$

In brief, my answers run as follows. On (1), I argue in $\$ 3$ of this paper that there exist significant differences regarding this debate as it occurs in the context of SR, versus as it occurs in GR. The principal reason for this is that, while the advocate of the dynamical approach may be regarded as seeking to ontologically reduce the metric field in theories with fixed metric structure (such as SR) to the symmetry properties of matter fields (cf. [10, 42]), she does not attempt to make such a move in theories with dynamical metric structure, such as GR ${ }^{1}$

\footnotetext{
${ }^{1}$ For further discussion regarding how this debate changes on moving from SR to GR,
} 
On (2), I argue in $\$ 4$ that it is important to distinguish between what I call 'qualified' versus 'unqualified' explanations in the context of this debate ${ }^{2}$ Once this distinction is made, the geometrical approach bifurcates into two positions, which I call, respectively, the 'qualified' and 'unqualified' geometrical approaches. In \$5, I argue that distinguishing between these two positions is crucial, for while the former version of the geometrical approach is tenable, the latter is not; nevertheless, these two views have been run together in much of the literature on this topic up to this point.

On (3), I argue in $\$ 5$ that, while the 'unqualified' version of the geometrical approach is distinct from the dynamical approach in the context both of theories such as SR and of theories such as GR, the 'qualified' geometrical approach, by contrast, is only distinct from the dynamical approach in the former context.

On (4), I argue that there is an important sense in which Knox's spacetime functionalism, according to which "the spacetime role is played by whatever defines a structure of local inertial frames" [32, p. 22], constitutes an extension of the dynamical approach - in essence stating that whichever structure has chronogeometric significance may be identified as playing the functional role of spacetime, and therefore, on a functionalist approach to the definition of physical quantities, may be identified as being spatiotemporal tout court $\mathrm{H}^{3}$ In addition, I argue that Weatherall in [69, §6] is most plausibly read as both (a) embracing spacetime functionalism, and (b) embracing either the dynamical or the 'qualified' geometrical approach.

Along the way, a number of other tasks are accomplished. Most notably, I demonstrate that what were labelled in [52, §5] two 'miracles' of GR - (1) that all dynamical laws for matter fields have the same local (Poincaré) symmetry properties; and (2) that these local

see 6, 10, 52. In light of the fact that the advocate of the dynamical approach does not attempt to undertake an ontological reduction of the above-described kind in the context of GR, one might be inclined to conclude: 'So much the worse for the dynamical approach in the context of GR, as a distinct view in the landscape'. Below, I will argue that there is something to this concern, for (I maintain) there is no difference in the GR context between the dynamical approach and the most defensible version of the geometrical approach.

${ }^{2}$ It is worth flagging that I will offer these two notions of explanation without claiming (or seeking) to give a full conceptual analysis of the notion of scientific explanation; in my view, the distinction between 'qualified' and 'unqualified' explanations is still a valuable and comprehensible one (providing, as I see it, at least some of the "explanatory concepts" which Norton suggests may be necessary for "a full understanding of constructivism [i.e., the dynamical approach]" [44, p. 824]), even in the absence of such an analysis. (In this regard, cf. the methodology of [70, pp. 15-16].)

${ }^{3} \mathrm{By}$ contrast, there is a sense in which advocates of the dynamical approach need not speak of 'spacetime' at all-cf. [10, §3.1]. 
symmetries coincide (in the relevant regime in which curvature effects may be ignored) with the symmetries of the ontologically autonomous metric field in the theory - admit of no resolution from within GR, on any plausible form of the dynamical or geometrical approaches, modulo some hints from recent work on the so-called 'geodesic principle' in GR regarding the second 'miracle' 4

\section{Background}

Before proceeding to the matters outlined above, I review in this section some standard discussion regarding (i) the formulation of classical spacetime theories (\$2.1); (ii) symmetries in such theories $(\$ 2.2)$; and (iii) presentations of special and general relativity $(2.3)$

\subsection{Spacetime theories}

Let us say-following e.g. [2, 48, 49, 59] - that the kinematically possible models (KPMs) of a given spacetime theory are picked out by tuples $\left\langle M, \Phi_{1}, \ldots, \Phi_{n}\right\rangle$, with (a) $M$ a (four-dimensional) differentiable manifold; and (b) the $\Phi_{1}, \ldots, \Phi_{n}$ various (tensor) fields on $M !^{5}$ Given a class of KPMs for a given theory, let us then say that the dynamically possible models (DPMs) of that theory are those KPMs the $\Phi_{1}, \ldots, \Phi_{n}$ of which satisfy certain specified dynamical equations.

To illustrate, consider two examples. First, take a special relativistic massless Klein-Gordon theory (call it KGS). In this theory, KPMs are triples $\left\langle M, \eta_{a b}, \varphi\right\rangle$, where $\eta_{a b}$ is a fixed Minkowski metric field on $M$ (fixed identically in all KPMs - see [49, p. 115]), and $\varphi$ is a real scalar field on $M$. DPMs of KGS are picked out as those KPMs the fields of which satisfy the massless Klein-Gordon equation $\sqrt{6}$

$$
\eta_{a b} \nabla^{a} \nabla^{b} \varphi=0 .
$$

\footnotetext{
${ }^{4}$ It is worth noting that these two 'miracles' of GR may admit of resolution in a successor theory to GR, in a manner analogous to that in which the 'miracle' of the coincidence of gravitational and inertial masses in Newtonian mechanics was resolved on moving to GR. See 61] for a detailed discussion of the explanation of the coincidence of gravitational and inertial masses in GR, and [51] for how the two 'miracles' of GR may be resolved on moving to one particular successor theory-viz., perturbative string theory.

${ }^{5}$ In principle, we should not exclude other types of field on $M$ - e.g. spinor fields; pseudotensors; tensor densities; etc. (For arguments for taking these latter two classes of object seriously, see [45, 46.) In this paper, however, I focus exclusively upon the case in which the $\Phi_{i}$ are tensor fields.

${ }^{6}$ Here, $\nabla_{a}$ is the torsion-free derivative compatible with $\eta_{a b}$, so that $\nabla_{a} \eta_{b c}=0$.
} 
As a second example, consider a general relativistic Klein-Gordon theory (call it KGG). In this case, KPMs are again triples $\left\langle M, g_{a b}, \varphi\right\rangle-$ this time, however, $g_{a b}$ is a generic Lorentizan metric field on $M$, not fixed in all DPMs of the theory. In this case, DPMs are picked out by the GR Klein-Gordon equation 7

$$
g_{a b} \nabla^{a} \nabla^{b} \varphi=0
$$

and the Einstein field equations 8

$$
G_{a b}=8 \pi T_{a b},
$$

where $T_{a b}$ is the stress-energy tensor associated with $\varphi \cdot 9$

\section{$2.2 \quad$ Symmetries}

I now draw a standard distinction between metric symmetries, and dynamical symmetries (cf. e.g. [18, §3.4]).

For a given metric field, let us say that a coordinate transformation is a metric symmetry (sometimes: an isometry) just in case the metric field is unaltered by the coordinate transformation. For example, the symmetries of the Minkowski metric field $\eta_{a b}$ of special relativity are the Poincaré transformations - those affine transformations 10

$$
x^{\mu} \rightarrow \Lambda_{\mu^{\prime}}^{\mu} x^{\mu^{\prime}}+a^{\mu}
$$

the linear transformation matrix components $\Lambda_{\mu^{\prime}}^{\mu}$ of which satisfy

$$
\Lambda_{\mu^{\prime}}{ }^{\mu} \Lambda_{\nu^{\prime}}{ }^{\nu} \eta_{\mu \nu}=\eta_{\mu^{\prime} \nu^{\prime}}
$$

and which are hence Lorentz transformations. By contrast, the metric field $g_{a b}$ of GR need not in general have any non-trivial symmetriesalthough it might, in particular models of the theory.

In addition to the notion of metric symmetries, it is useful to introduce the notion of a dynamical symmetry. A coordinate transformation is a dynamical symmetry just in case the dynamical equations governing non-gravitational fields take the same form in coordinate

\footnotetext{
${ }^{7}$ The torsion-free derivative operator $\nabla_{a}$ now compatible with $g_{a b}$, so that $\nabla_{a} g_{b c}=0$.

${ }^{8}$ These are the Einstein field equations with vanishing cosmological constant $\Lambda$. For $\Lambda \neq 0$, the field equations read $G_{a b}+\Lambda g_{a b}=8 \pi T_{a b}$.

${ }^{9}$ Recall that the stress-energy tensor is defined through $T^{a b}:=\frac{2}{\sqrt{g}} \frac{\delta S}{\delta g_{a b}}$, where $g$ is the metric determinant, and $S$ is the action to which the matter Lagrangian - here $\mathcal{L}_{\mathrm{EM}}$ - is associated. $T_{a b}$ is defined from $T^{a b}$ via $T_{a b}:=g_{a c} g_{b d} T^{c d}$.

${ }^{10}$ Here, I switch to a coordinate-based description-hence the transition from Roman (abstract) to Greek indices.
} 
systems related by that transformation ${ }^{11}$ For example, transforming the SR Klein-Gordon equation (1) from one coordinate system to a second via an affine transformation 12

$$
\begin{aligned}
\eta_{\mu \nu} \nabla^{\mu} \nabla^{\nu} \varphi & =0 \\
\longrightarrow \quad \eta_{\mu \nu} M_{\mu^{\prime}}^{\mu} M_{\nu^{\prime}}^{\nu} \nabla^{\mu^{\prime}} \nabla^{\nu^{\prime}} \varphi & =0,
\end{aligned}
$$

one finds that such an equation is invariant under the transformation when (5) is satisfied - i.e. if the affine transformation is a Poincaré transformation. Thus, the dynamical symmetries associated with (1) at least include the Poincaré transformations.

\subsection{Special and general relativity}

Having introduced the necessary details regarding spacetime theories and their symmetries, in this section I characterise - with both greater precision and generality — what it means for a given theory to be 'special relativistic' (2.3.1), versus 'general relativistic' (2.3.2).

\subsubsection{Special relativity}

In this paper, I take special relativistic theories to be characterised by the following two criteria:

- KPMs at least include a fixed Minkowski metric field $\eta_{a b}$-so may be written $\left\langle M, \eta_{a b}, \Phi_{1}, \ldots, \Phi_{n}\right\rangle$.

- DPMs are picked out by the requirement that dynamical equations for the $\Phi_{1}, \ldots, \Phi_{n}$ be Poincaré invariant.

\footnotetext{
${ }^{11}$ In this paper, I mean by 'matter fields', or 'non-gravitational fields', those for which there exists an associated stress-energy tensor, and by 'gravitational fields' those for which there exists no such stress-energy tensor-this distinction is in the spirit of [34]. In the context of GR, this means that the metric field is identified as a gravitational field, whereas all other fields typically of interest (e.g. Klein-Gordon fields, electromagnetic fields, etc.) are matter fields. Clearly, there exist subtle issues here regarding the possibility of defining a stress-energy tensor associated with the metric field in GR - see 15] for a proof against this possibility, and e.g. [25, 33, 50, for related discussion. Note also that this distinction between matter and gravitational fields may break down in the case of other spacetime theories - for example, in Newtonian gravitation theory (cf. \$5.2.2), it is possible to define a stress-energy tensor associated with the potential $\varphi$, in spite of this field naturally being regarded as 'gravitational' (cf. [17]). Nevertheless, for my purposes, the above distinction will suffice.

${ }^{12}$ Here, again, I use a coordinate-based description; in this case the linear transformation matrix components in this affine transformation are written $M_{\mu^{\prime}}^{\mu}$, for reasons which shall become clear. Note that I do not transform the fixed fields - cf. [49, p. 115].
} 
The latter criterion is referred to by Brown as the big principlesee e.g. [6, §8.4.1] ${ }^{13}$ Note that, by construction, metric and dynamical symmetries coincide in special relativistic theories.

\subsubsection{General relativity}

Turn now to the question of what it is for a spacetime theory to be general relativistic. For the purposes of this paper, I take such theories to be characterised by the following two criteria:

- KPMs at least include a Lorentzian metric field $g_{a b}$ - so may be written $\left\langle M, g_{a b}, \Phi_{1}, \ldots, \Phi_{n}\right\rangle$.

- DPMs are picked out by dynamical equations for the $\Phi_{1}, \ldots, \Phi_{n}$, along with the Einstein field equations $G_{a b}=8 \pi T_{a b}$, where $T_{a b}$ is the stress-energy tensor for the $\Phi_{1}, \ldots, \Phi_{n}$.

This characterisation of a general relativistic theory is very weaknote, in particular, that there is no guarantee in general relativistic theories so understood that metric symmetries coincide locally with dynamical symmetries (as was the case for special relativistic theories, as presented above) ${ }^{14}$ Such requirements may be imposed via restriction to those models of GR which satisfy further conditions; from the point of view of the matter of symmetry coincidence, one particular auxiliary condition which will be of significance is ${ }^{15}$

- Instantiation of the strong equivalence principle (SEP).

Whence this third assumption? What exactly is the SEP, and why need it be imposed in one's characterisation of a general relativistic theory? A full answer to these questions will require some detailed discussion.

The SEP is intended to capture facts regarding the 'local validity' of SR in GR. Brown puts the point thus ${ }^{16}$

\footnotetext{
${ }^{13}$ In his 1908 paper [40], Minkowski referred to this principle as the world-postulate-for discussion, see [6, §8.1].

${ }^{14}$ One further observation about the distinction between special versus general relativistic theories as characterised above: Since the metric field $\eta_{a b}$ of SR is fixed identically in all KPMs, so too is the manifold $M$ on which that field is defined. Not so for GR: since it is not definitional of a general relativistic theory that it contain a certain fixed field, there may exist models with distinct manifolds $M$.

${ }^{15}$ Other conditions which one may be interested in imposing upon the class of GR solutions in which one is interested are e.g. energy conditions, for such conditions are often understood to be tied to the restriction to 'physically reasonable' matter (for example, to conditions that energy cannot be negative). For a recent virtuoso study of energy conditions, see 14 .

${ }^{16}$ Other similar presentations of the SEP can be found in e.g. [30, §3.4] and [31, p. 874].
} 
There exists in a neighbourhood of each event preferred coordinates, called locally inertial at that event. For each fundamental non-gravitational interaction, to the extent that tidal gravitational forces can be ignored, the laws governing the interaction find their simplest form in these coordinates. This is their special relativistic form, independent of spacetime location. [6, p. 169]

Here, there exist a number of subtleties regarding what is meant by the qualification "to the extent that tidal gravitational forces can be ignored", and moreover regarding whether other foundational principles GR - for example minimal coupling, which is a heuristic prescription for the construction of dynamical laws for non-gravitational fields in GR from those in SR - are compatible with the SEP as formulated above. Since these matters are not directly relevant to my purposes in this paper, I refer the reader to [9, 10, 52 for detailed discussion. For today, the essential aspect of the SEP is the imposition that, in the neighbourhood of any $p \in M$ in GR, laws of physics recover their 'special relativistic form'-where I shall understand this to mean: a Poincaré invariant form. Clearly, this is a particular restriction on the matter sector in the theory.

To illustrate, consider again the general relativistic Klein-Gordon equation (2). Written in an arbitrary coordinate basis, this reads

$$
g_{\mu \nu} \partial^{\mu} \partial^{\nu} \varphi+\Gamma_{\nu \mu}^{\mu} \partial^{\nu} \varphi=0 .
$$

Recall that in a coordinate basis $\left\{e_{\mu}\right\}$, the connection components $\Gamma_{\nu \rho}^{\mu}$ associated to a derivative operator $\nabla_{a}$ are defined by $\nabla_{\rho} e_{\nu}=: \Gamma_{\nu \rho}^{\mu} e_{\mu}$. Then, at any $p \in M$, we can choose normal coordinates, such that $\Gamma_{(\nu \rho)}^{\mu}(p)=0$ in those coordinates; for a torsion-free derivative operator, we can in fact choose normal coordinates such that $\Gamma_{\nu \rho}^{\mu}(p)=0$. (Note that the connection components away from $p$ will in general not vanish.) If the unique torsion-free, metric compatible derivative operator is used, then in normal coordinates we also have $g_{\mu \nu, \rho}(p)=0$, and one may further restrict to the subset of normal coordinates at $p$ such that $g_{\mu \nu}(p)=\operatorname{diag}(-1,1,1,1)$. Since $g_{\mu \nu}(p)$ takes this diagonal form - preserved under Poincaré transformationsin this restricted class of normal coordinates at $p$, one might write $g_{\mu \nu}(p)=\eta_{\mu \nu}$ (cf. e.g. [41, p. 1055]). This notwithstanding, however, any claim to the effect that the metric field 'reduces' to the the Minkowski metric at $p$ in normal coordinates should be met with suspicion - for in general, second (and higher) order derivatives of the metric field do not vanish at $p$, in these coordinates.

In normal coordinates at $p \in M,(2)$ (in a coordinate basis, (8)) takes a particularly simple form at $p$ : 


$$
\eta_{\mu \nu} \partial^{\mu} \partial^{\nu} \varphi=0
$$

moreover, this form (with the metric field diagonalised) is retained in all frames related by Poincaré transformations. This illustrates the sense in which certain dynamical equations for non-gravitational fields recover locally a Poincaré invariant form. That all dynamical laws for non-gravitational fields in GR manifest this quality is a statement of the SEP. Importantly, note that, absent the imposition of the SEP, it is not the case that all dynamical equations for matter fields in GR need be locally Poincaré invariant. For example, there exists no a priori prohibition on the existence of matter fields obeying dynamical laws which are locally Galilean invariant, in a spacetime theory with a dynamical, Lorentzian metric field satisfying the Einstein field equations 17

Why should one restrict to those solutions of GR in which the SEP is satisfied? The reason is that this principle - which ensures that, locally, the (Poincaré) symmetries of the metric field 18 coincide with the (Poincaré) symmetries of the dynamical laws governing matter fields - is typically regarded to constitute an important condition for the chronogeometricity of the metric field - that is, for intervals as given by the metric field to be read off by stable rods and clocks built from matter fields. As Brown writes 19

It is because of ... local Lorentz covariance that rods and clocks, built out of the matter fields which display that symmetry, behave as if they were reading aspects of the metric field and in so doing confer on this field a geometric meaning. That light rays trace out null geodesics of the field is again a consequence of the strong equivalence principle, which asserts that locally Maxwell's equations of electrodynamics are valid. [6, p. 176]

That is, the SEP is, it is argued, an important condition for the

\footnotetext{
${ }^{17}$ See [30, 51] for discussion of this possibility.

${ }^{18}$ Again, modulo subtle issues regarding the qualification "to the extent that tidal gravitational forces can be ignored" - see [9, 10, 52, for discussion.

${ }^{19}$ One might reasonably pause over whether the "as if" in the following passage is necessary, on Brown's account.
} 
metric field $g_{a b}$ in GR to have (local) operational meaning ${ }^{20}{ }^{21}$ I return to discuss further the SEP, in light of the so-called geodesic principle in $\mathrm{GR}$, in $\$ 6$.

\section{The dynamical/geometrical debate}

The above in hand, in this section I demonstrate how the dynamical/geometrical debate plays out in the context of both SR (\$3.1) and GR ( 3.2$){ }^{22}$ a detailed reconsideration of the geometrical approach will follow in $\S ₫ 45$. At the most general level, the dynamical/geometrical debate centres upon the following question:

Whence the metric field's chronogeometric significance?

Taking, as elaborated above, the (local) coincidence of metric and dynamical symmetries to be an important condition which must be fulfilled in one important means via which the metric field acquires its chronogeometric significance (viz., via the SEP), one antecedent question which one might seek to address in order to answer the above is the following 23

Why do metric symmetries coincide (locally) with dynamical symmetries?24

\footnotetext{
${ }^{20}$ One might wonder whether satisfaction of the SEP should be regarded as being a necessary condition for the metric field to have its chronogeometric significance, or rather as being a sufficient condition, or rather something else. In [52, pp. 15-16], it is indeed claimed that the SEP constitutes a necessary condition for chronogeometricity; however, it is perhaps more conservative to state that, with auxiliary assumptions such as the existence of stable rods and clocks, it constitutes a jointly sufficient condition for chronogeometricity. In this way, one does not rule out other possible means of gaining operational access to the metric field - for example, by using test particles which traverse null and timelike geodesics to gain access to conformal and projective structure, from which (via Weyl's theorem-type reasoning - cf. [20, 72, 73, and with certain further additional assumptions) one can recover metric structure. There remains much further work to be done in order to understand fully these alternative means of gaining operational access to the metric field; cf. footnote 55 for some further discussion of Weyl's theorem, and [11, §4] for related discussion.

${ }^{21}$ Similarly, one might argue that postulating that metric symmetries coincide with dynamical symmetries in SR is an important condition for the metric field $\eta_{a b}$ to have operational meaning in that case.

${ }^{22}$ There is a sense in which the lessons of $\S \$ 3.1$ and 3.2 can be generalised to all theories with, respectively, fixed versus dynamical metric structure - see [10, §5].

${ }^{23}$ For the time being, my focus is on this mode of gaining operational access to the metric field - though I concede that there may be other means, as discussed in footnote 20 above, and in \$6 below.

${ }^{24}$ The 'locally' qualification is of particular significance in GR, since the SEP ensures
} 
It is upon this latter question that much of the dynamical/geometrical debate has focussed. Prima facie, advocates of the dynamical approach (developed in particular by Brown and Pooley [6, 7, 8]) appear to offer very different accounts of this coincidence of symmetries to advocates of the geometrical approach (for example, Friedman or Maudlin [22, 38]). In the remainder of this section, I discuss the dynamical/geometrical debate in the context of both SR (\$3.1) and GR $(\$ 3.2)$.

\subsection{Special relativity}

In this subsection, I consider the account of the coincidence of metric and dynamical symmetries in SR proffered on the part of advocates of the geometrical (3.1.1) and dynamical (\$3.1.2) approaches.

\subsubsection{The geometrical approach}

Why, in special relativistic theories, do dynamical symmetries coincide with symmetries of the Minkowski metric field $\eta_{a b}$ ? Advocates of the geometrical approach to spacetime theories seek to answer this question via some appeal to $\eta_{a b}$ itself. To be specific, in this paper I focus upon a version (later: versions) of the approach according to which the Minkowski metric field $\eta_{a b}$ of SR is ontologically autonomous and primitive, and (somehow; in some sense to be cashed out) constrains the possible form of dynamical equations for matter, such that metric symmetries coincide with dynamical symmetries. As Maudlin writes, [38, pp. 117-8]

... the Minkowski geometry takes exactly the same form described in [any] Lorentz coordinate system (by the symmetry of Minkowski spacetime), and the laws of physics take exactly the same coordinate-based form when stated in a coordinate-based language in any Lorentz coordinate system (because the laws can only advert to the Minkowski geometry, and it has the same coordinate-based description). (My emphasis.)

That a notion of constraint is at play on this view is manifest in the italicised portion of the above quotation. While advocates of the dynamical approach often object that such a notion of constraint or explanation is mysterious - for example, Brown writes "It is wholly unclear how this geometrical explanation is supposed to work." [6,

the local coincidence of metric and dynamical symmetries, in the neighbourhood of a given point $p \in M$. 
p. 134] - I will assess in $\$ 4$ the extent to which such objections find their mark. In the meantime, I turn to the dynamical approach to SR.

\subsubsection{The dynamical approach}

The dynamical approach offers a very different perspective on the coincidence of metric and dynamical symmetries in SR. According to this view, the metric field $\eta_{a b}$ is not ontologically autonomous and primitive; rather, it is a codification of the symmetry properties of the dynamical equations governing matter fields. (One may, therefore, understand the dynamical approach to SR - and to theories with fixed metric structure more generally - as an ontological thesis; as a form of relationalism - cf. [48, §6.3.2].) As Brown puts it: (Cf. also [8, p. 80].)

The appropriate structure is Minkowski geometry precisely because the laws of physics of the non-gravitational interactions are Lorentz covariant. [6, p. 133]

In other words (those of Myrvold), on the dynamical view,

[T] he connection between spacetime [metric] structure and dynamical symmetries and asymmetries is analytic. [42, p. 13]

If such a view regarding the analytic connection between metric and dynamical symmetries can be made to hold together, then that metric and dynamical symmetries coincide in SR follows automatically; in this way, a straightforward account of this coincidence is, apparently, available.

The question of whether the dynamical approach to SR is viable has been widely discussed - see e.g. [1, 6, 26, 29, 44, 48, 56, 57]. In this paper, I focus on a different issue: whether advocates of the dynamical approach have been fair to the geometrical approach, and whether 'geometricians' can, in fact, offer a coherent answer to the question of why metric and dynamical symmetries coincide, in SR. Before doing so, however, I consider how the nature of the dynamical/geometrical debate shifts on moving to GR.

\subsection{General relativity}

In the GR context, advocates of both the dynamical and geometrical approaches agree that the metric field $g_{a b}$ is an ontologically autonomous entity, obeying its own dynamical equations, and not straightforwardly reducible to (symmetries of dynamical equations 
governing) matter fields, as per the dynamical approach to SR. ${ }^{25}$ However, the two approaches prima facie continue to issue different verdicts on the question of why metric and dynamical symmetries may be taken (locally) to coincide. In this subsection, I review the geometrical (3.2.1) and dynamical (\$3.2.2) approaches to GR.

\subsubsection{The geometrical approach}

Advocates of the version (later: versions) of the geometrical approach to GR under consideration in this paper maintain that, locally in the neighbourhood of any $p \in M$ (and in the regime in which 'tidal gravitational forces' may be ignored - cf. \$2.3.2), metric and dynamical symmetries coincide (in accordance with the SEP), because the metric field $g_{a b}$ (somehow; in some sense to be cashed out) constrains the possible form of dynamical equations for matter, such that metric symmetries coincide (locally) with dynamical symmetries. While, again, the advocate of the dynamical approach may find the notion of constraint here mysterious, I discuss in $\S \$ 4$ the extent to which these matters can be accounted for by advocates of the geometrical approach.

\subsubsection{The dynamical approach}

Assuming that the metric field in GR is not ontologically reducible to (symmetries of dynamical laws governing) matter fields, the foregoing (cf. 33.1 .2 proffered explanation on the part of advocates of the dynamical approach to SR cannot be applied in the GR context. Thus, for the advocate of the dynamical approach, there are two brute facts in GR - two conspiracies, or 'miracles', which lack further explanation from within the theory - whereas in SR there is only one (see below) ${ }^{26}$

MR1: All non-gravitational interactions are locally governed by Poincaré invariant dynamical laws.

MR2: The Poincaré symmetries of the dynamical laws governing non-gravitational fields in the neighbourhood of any point $p \in M$ coincide (in the regime in which terms representing 'tidal gravi-

\footnotetext{
${ }^{25}$ There exist significant difficulties regarding attempts to tell such a story of ontological reduction in GR; an obvious illustration can be found in the existence of vacuum solutions in the theory. This said, the question of whether an ontological excision of the metric field in GR is possible remains of philosophical and conceptual interest-particularly to advocates of the dynamical approach, for whom this would afford a means of bringing their approach to GR into line with their approach to SR.

${ }^{26}$ See [52, §5], where the terminology of 'miracles' was introduced, for further discussion.
} 
tational forces' can be ignored) with the symmetries of the metric field in that neighbourhood.

There are two points to make here. First, note that MR1 held also in SR: that all non-gravitational interactions are (locally) governed by Poincaré invariant dynamical laws is a brute fact - an outset assumption - in both theories, which (the advocate of the dynamical approach contends) admits of no further explanation from within each theory. Second, as I argue in $\$ 5.3$, while an untenable form of the geometrical approach may purport to account for both MR1 and MR2, any acceptable form of the geometrical approach must also accept these two miracles of GR. In this sense, the existence of these two miracles is independent of the dynamical/geometrical debate.

\section{Qualified and unqualified explana- tions}

In $\$ 5$, I consider whether the advocate of the geometrical approach has received an unduly rough ride in the recent philosophical literature. Essentially, my answer will be affirmative, because an untenably strong form of the geometrical approach has constituted the target of e.g. 6, 8, 9, 52, In order to make these points, however, I must first distinguish between what I call qualified versus unqualified explanations:

- (Qualified explanations.) Consider one particular dynamical equation featuring coupling to a metric field - for example, the special relativistic Klein-Gordon equation (1), or the general relativistic Klein-Gordon equation (2). Then ask: might the metric field in the theory in question $\left(\eta_{a b}\right.$ in the case of KGS; $g_{a b}$ in the case of KGG) feature in an explanation of the form (in particular, of the symmetries) of that dynamical equation, and of the behaviour of the matter field(s) (here $\varphi$ ) to which it is coupled? Call this the question of qualified explanation - for the concern here is with accounting for the form of one, given dynamical equation, and for the behaviour of the particular fields coupled in that equation.

- (Unqualified explanations.) Consider all possible dynamical equations consistent with a given theory, such as SR or GR. ${ }^{27}$ Then

\footnotetext{
${ }^{27}$ There is some ambiguity regarding what is meant by a 'theory' here. To be clear, by 'theory' is meant here a theoretical framework such as that for SR or GR as presented in 2.3. rather than specific theories within those frameworks, such as KGS or KGG.
} 
ask: does the metric field in the theory in question $\left(\eta_{a b}\right.$ in the case of SR; $g_{a b}$ in the case of GR) explain the form (in particular, the symmetries) of all those possible equations consistent with the theory, and (in a certain particular way to be articulated) the behaviour of all possible matter fields, such that assumptions made in the formulation of the theory regarding the form of those equations and the behaviour of matter fields (e.g., that massless particles in GR traverse null geodesics) are, ultimately, redundant? For example, can $\eta_{a b}$ explain the fact that all dynamical laws governing matter fields in SR are Poincaré invariant, or can $g_{a b}$ in GR explain the SEP? Call this the question of unqualified explanation.

\section{The geometrical approach}

In this section, the above distinction between qualified and unqualified explanations is brought to bear on the question of whether there exists any viable form of the geometrical approach. My answer will be the following: while the form of the geometrical approach considered in e.g. [6, 8, 9, 52 is not viable, there exists a weaker version of the approach, which can be defended.

The section proceeds as follows. In \$5.1. I distinguish between these two versions of the geometrical approach, before exploring the different accounts they give regarding the role of the metric field in explanations of the coincidence of (local) metric and dynamical symmetries, and of the behaviour of matter fields to which they couple, in both SR (\$5.1.1) and GR (\$5.1.2). In $\$ 5.2$, I explore some further consequences of what I take to be the more defensible version of the geometrical approach. In $\$ 5.3$, I demonstrate that this version of the geometrical approach does not account for MR1 and MR2.

\subsection{Two geometrical approaches}

The geometrical approach, in both SR (\$3.1.1) and GR (\$3.2.1), may be understood in (at least) two different ways. Drawing upon the distinction presented in $\$ 4$, the versions of the approach that I consider in this paper are dubbed the qualified versus unqualified geometrical approaches:

- (Qualified geometrical approach.) Consider a particular dynamical equation governing the behaviour of a particular set of nongravitational fields $\Phi_{1}, \ldots, \Phi_{n}$. Insofar as that equation features coupling to a metric field (as in e.g. (1) in KGS, or (2) in KGG), 
that metric field may contribute to an explanation of the symmetries of that dynamical equation, and of the dynamical behaviour of those $\Phi_{1}, \ldots, \Phi_{n}$ fields.

- (Unqualified geometrical approach.) Consider the metric field associated with a particular theory (for example, $\eta_{a b}$ in SR, or $g_{a b}$ in GR). That metric field constrains the form of all possible dynamical laws for non-gravitational fields consistent with that theory, such that assumptions about (local) dynamical symmetries are redundant in the formulation of the theory, and such that certain facts about the behaviour of matter fields are fixed.

In the following, I abbreviate 'the qualified geometrical approach' to QGA, and 'the unqualified geometrical approach' to UGA. On QGA, a particular metric field coupling to a particular set of nongravitational fields in a particular dynamical equation may be understood to contribute to a qualified explanation (in the sense in 84 ) of the symmetries of that dynamical equation, and of the dynamical behaviour of those non-gravitational fields. On UGA, a particular metric field is taken to explain the symmetries of all possible dynamical equations in a given theory, and to fix certain facts about the behaviour of all possible matter fields, such that we need not, in fact, make any assumptions regarding dynamical symmetries, or about those dynamical facts, in that theory. (Importantly, I take both QGA and UGA to maintain the ontological autonomy of the metric field in both SR and GR.) It is principally UGA which is attacked in [6, 8, 9, 52, and it is this version of the geometrical approach which is (I maintain) untenable.

\subsubsection{Special relativity}

The reasons why UGA is untenable are similar in both the SR and GR cases; I begin with the former. The worry regarding UGA is put clearly by Brown and Pooley: [8, p. 84]

As a matter of logic alone, if one postulates spacetime structure as a self-standing, autonomous element in one's theory, it need have no constraining role on the form of the laws governing the rest of the theory's models. So how is its influence supposed to work? Unless this question is answered, spacetime cannot be taken to explain the Lorentz covariance of the dynamical laws.

The point here is that it is consistent to have dynamical laws for non-gravitational fields in a theory featuring a Minkowski metric field $\eta_{a b}$, which nevertheless do not manifest the Poincaré symmetries of 
that metric field. As a concrete example, consider a modified version of KGS - call it LAS - KPMs of which are quadruples $\left\langle M, \eta_{a b}, \delta_{a b}, \varphi\right\rangle$, where $\delta_{a b}$ is a four-dimensional fixed Euclidean metric field ${ }^{28}$ and DPMs of which are picked out by the four-dimensional Laplace equation (hence my chosen nomenclature) ${ }^{29}$

$$
\delta_{a b} \nabla^{a} \nabla^{b} \varphi=0 .
$$

The dynamical symmetries of (10) do not include the Poincaré transformations (as for (1)); rather, they include the Euclidean transformations: those affine transformations the linear transformation matrix of which satisfies (cf. (5))

$$
M_{\mu^{\prime}}{ }^{\mu} M_{\nu^{\prime}}{ }^{\nu} \delta_{\mu \nu}=\delta_{\mu^{\prime} \nu^{\prime}}
$$

LAS illustrates that a theory's featuring a certain metric field in its KPMs is insufficient for that theory's dynamical equations for nongravitational fields to manifest the symmetries of that metric field, or for that metric field to play any constraining role in the dynamics of the matter fields in that theory, for those non-gravitational fields may couple to other fields (in this case, $\delta_{a b}$ ), such that metric symmetries and dynamical symmetries do not coincide, and such that the matter fields manifest other dynamical behaviour (than that which they would manifest if they were coupled to the metric field under consideration, here $\left.\eta_{a b}\right)$. Of course, one may wish to exclude coupling to such other fields; however, note that we then return to the situation in which the dynamical equations for matter fields manifesting certain symmetries, and yielding certain behaviour for those matter fields (e.g., that massless particles propagate on null geodesics), is an input assumption - it does not follow from (e.g.) $\eta_{a b}$ alone.

On the other hand, QGA faces no such problems, for in this case the concern is not with generic, unqualified claims, but rather with the form of one particular dynamical equation and with the dynamical behaviour of the matter fields coupled in that equation. Why is (1) Poincaré invariant? Because it features coupling to the $\eta_{a b}$ fieldcf. $\sqrt{2.2}$. Why is (10) Euclidean invariant? Because it features coupling to $\delta_{a b}$. Changing $\eta_{a b}$ in (1) to $\delta_{a b}$ in (10) changes the behaviour of $\varphi$ accordingly (after all, it is now governed by a different dynamical equation) - and it is very plausible to regard this as constituting a legitimate (if partial, for other factors may also be relevant to the

\footnotetext{
${ }^{28}$ The notation $\delta_{a b}$ is chosen to emphasise the analogy with the Kronecker delta $\delta^{a}{ }_{b}$; strictly, however, these are different objects, and should not be confused.

${ }^{29}$ Note that 10 is simply 1 , with $\eta_{a b}$ replaced by $\delta_{a b}$; in making this move, the dynamical equation becomes an elliptic, rather than hyperbolic, partial differential equation.
} 
dynamics of the field in question) explanation of the behaviour of $\varphi$. Thus, I take it that, in SR (and indeed, in the context of theories with fixed metric structure more generally), it is incorrect to regard as viable the explanation for the dynamical behaviour of matter proffered on the part of advocates of UGA, but correct to so regard the explanations proffered on the part of advocates of QGA. I discuss QGA further in 55.2 .

\subsubsection{General relativity}

Similar points to those made above apply in the case of GR. According to advocates of UGA, the metric field $g_{a b}$ in GR accounts for the local behaviour of all non-gravitational fields, such that the assumption of the SEP in the presentation of general relativistic theories in $\$ 2.3 .2$ is redundant, and such that matter fields must exhibit certain behaviour (e.g., such that test particles propagate on null geodesics). However, against such a claim, problem cases may also be identified.

In parallel with our introduction of LAS in \$5.1.1, I present here one such theory: the Jacobson-Mattingly theory (introduced in e.g. [12, $27]^{30}$, in which the action for a coupled Einstein-Maxwell system is augmented with an additional term (via a Lagrange multiplier field $\lambda$ ), imposing (as a field equation, via variation with respect to $\lambda$ ) that the vector potential $A^{a}$ be locally timelike 31

$$
S_{\mathrm{JM}}\left[g_{a b}, A^{a}, \lambda\right]=\int d^{4} x \sqrt{-g}\left(R-\frac{1}{4} F^{a b} F_{a b}+\lambda\left(g_{a b} A^{a} A^{b}-1\right)\right) .
$$

The imposition of this Lagrange multiplier term means that, in this theory, the dynamical behaviour of non-gravitational fields does not reflect the local (Poincaré) symmetries of the metric field. Rather, the (local) symmetries of the dynamical laws are a proper subset of the (local) metric symmetries. Given this, however, we appear to have in our possession a problem case for UGA, according to which the metric field constrains dynamical equations to manifest its own symmetries.

As with LAS in the case of SR, such cases appear to find their mark against UGA, for in the Jacobson-Mattingly theory, metric symmetries manifestly do not coincide with dynamical symmetries - so how

\footnotetext{
${ }^{30}$ In fact, the version of the Jacobson-Mattingly theory discussed here is a special case of that presented in $[12,27$.

${ }^{31}$ The first term is the Einstein-Hilbert action; $F_{a b}$ is the Faraday tensor associated to $A^{a}$. In this paper, I take it that in GR (or, as here, the Jacobson-Mattingly theory) a vector $\xi^{a}$ at a point is timelike just in case $g_{a b} \xi^{a} \xi^{b}>0$.
} 
could $g_{a b}$ be constraining the local form of dynamical equations in this strong sense? On the other hand, QGA again does not appear to face such problems. For example, consider (2) - as in the SR context, it is perfectly reasonable to claim that the coupling in this equation of $\varphi$ to $g_{a b}$ offers an explanation of the dynamical behaviour of $\varphi$; moreover, the fact that no generic, unqualified claim is made regarding possible form of dynamical equations for non-gravitational fields means that cases such as the Jacobson-Mattingly theory do not find their mark against QGA (for further discussion, see $\$ 5.2 .2$ ).

\subsection{The qualified geometrical approach}

I have argued that QGA is a defensible version of the geometrical approach, whereas UGA is not. In this subsection, I explore some further consequences of QGA. Specifically, I consider in 5.2 .1 the sense in which the metric field in a given theory may, in fact, be understood to account for the form of all dynamical laws in that theory. In $\$ 5.2 .2$. I consider whether an account of the dynamical behaviour of matter in terms of metric structure is available on QGA, even in problematic cases such as those described above, in which (local) metric symmetries do not coincide with (local) dynamical symmetries ${ }^{32}$ I close in 55.2 .3 by drawing a more fine-grained distinction within QGA.

\subsubsection{Univocal explanation}

In both SR and GR, there is a sense in which, on QGA, the metric field can explain the form of all dynamical laws in the theoryonce the restriction to a certain form of dynamical equations is made. For example, given the restriction in SR to dynamical equations for non-gravitational fields which take a Poincaré invariant form, we may write all such equations in coordinate-free notion featuring coupling to $\eta_{a}{ }^{33}$ in which case, $\eta_{a b}$ may feature in explanations of the dynamical behaviour of the matter fields under consideration. This does not explain the initial restriction to Poincaré invariant dynamical laws for non-gravitational fields, but it does mean that $\eta_{a b}$ may feature in explanations for the behaviour of all matter fields, once such an assumption is made. Similarly in GR, the metric field $g_{a b}$ may not be able to account for the initial restriction to dynamical equations for matter fields obeying the SEP, but it may feature in explanations of

\footnotetext{
${ }^{32}$ Strictly, I will have to generalise the notion of a 'metric symmetry' in $\$ 5.2 .2$, to account for the examples given in that section. This, however, will be of no consequence.

${ }^{33}$ Cf. [9, §5].
} 
the form of all dynamical laws for non-gravitational fields in GR, once this assumption is made-for in making this assumption, it is natural to consider dynamical equations in which matter fields are coupled to this very $g_{a b}$ field 34

\subsubsection{Partial explanation}

A further subtlety regarding QGA pertains to the issue of partial explanation. I make the following claim: even in the cases in which metric and dynamical symmetries do not coincide, the metric field may feature in explanations of the dynamical behaviour of matter, on QGA 5 To see this, it is useful to consider three sub-cases: (i) situations in which dynamical symmetries form a proper subset of metric symmetries; (ii) situations in which dynamical symmetries form a proper superset of metric symmetries; (iii) cases where dynamical symmetries partially overlap with metric symmetries.

In order to discuss each of these cases, it is useful to introduce here three versions of Newtonian gravitation theory (NGT). First, let a Leibnizian structure be a triple $\left\langle M, t_{a b}, h^{a b}\right\rangle$, where $M$ is a fourdimensional differentiable manifold; $t_{a b}$ is a fixed temporal 'metric' field on $M$ of signature $(1,0,0,0)$; and $h^{a b}$ is a fixed spatial (inverse) 'metric' field on $M$ of signature $(0,1,1,1) \sqrt{36}$ The $t_{a b}$ and $h^{a b}$ fields are orthogonal, so that

$$
h^{a b} t_{b c}=0
$$

furthermore, I restrict in this paper to structures (Leibnizian or otherwise; see below) which are temporally orientable, so that there exists

\footnotetext{
${ }^{34}$ It is worth making two related points here. (1): Technically, such coupling is not essential, for we might instead couple to e.g. a fixed Minkowski metric field $\eta_{a b}$, or to a generic Lorentzian metric field which satisfies not the Einstein field equations, but some other set of dynamical equations. In the cases in which all dynamical laws feature coupling to $g_{a b}$, however, this metric field may feature in explanations of the form of all these laws. (2): One need not make the assumption that all dynamical laws manifest certain (local) symmetries so explicitly - one might instead make assumptions of (e.g.) universal coupling of the metric field to matter fields in all dynamical equations for the latter; this may, then, entail the relevant facts about the symmetries of those laws. This, indeed, appears to be Maudlin's stance, when he writes that "the fundamental requirement of a relativistic theory is that the physical laws should be specifiable using only the relativistic space-time geometry. For Special Relativity, this means in particular Minkowski space-time." 38, p. 117] The point here is that, on QGA, one may appeal to the metric field in giving certain generic explanations of the behaviour of matter fields in a certain restricted class of models of the theory - but the metric field itself does not account for those restrictions.

${ }^{35} \mathrm{I}$ am grateful to Oliver Pooley for impressing this point upon me.

${ }^{36}$ Scare quotes are included on 'metric' here, for strictly neither $t_{a b}$ nor $h^{a b}$ satisfies the metric non-degeneracy condition - cf. [37, §4.1].
} 
a continuous (globally defined) one-form $t_{a}$ that satisfies the decomposition condition $t_{a b}=t_{a} t_{b}$ at every point [37, p. 251].

In contrast with the notion of a Leibnizian structure, let a Galilean structure be a quadruple $\left\langle M, t_{a b}, h^{a b}, \nabla_{a}\right\rangle$, consisting of a Leibnizian structure, together with a derivative operator $\nabla_{a}$ on $M$ satisfying the compaibility conditions

$$
\begin{aligned}
\nabla_{a} t_{b c} & =0, \\
\nabla_{a} h^{b c} & =0 .
\end{aligned}
$$

Finally, let a Newtonian structure be a tuple $\left\langle M, t_{a b}, h^{a b}, \nabla_{a}, \sigma^{a}\right\rangle$, consisting of a Galilean structure, together with a fixed vector field $\sigma^{a}$ on $M$, such that

$$
t_{a b} \sigma^{b} \neq 0 .
$$

Since none of Leibnizian, Galilean, or Newtonian structures are themselves metric fields, the notion of a metric symmetry cannot be applied in these cases ${ }^{37}$ However, the relevant notion easily generalises to the structures now under consideration: I say that a coordinate transformation is a structure symmetry just in case the structure under consideration is invariant under that transformation. Applying such a notion to Leibnizian, Galilean, and Newtonian structures, one finds that their associated structures symmetries are given by (no surprise!) the Leibniz, Galilean, and Newton groups ${ }^{38}$

With these three structures in hand, we can consider three different theories - viz., Newtonian gravitation theory set in each of these three structures. Consider first Newtonian mechanics set in a Galilean structure ${ }^{39} \mathrm{KPMs}$ of this theory are tuples $\left\langle M, t_{a b}, h^{a b}, \nabla_{a}, \varphi, \rho\right\rangle$, where $\varphi$ and $\rho$ are real scalar fields on $M$, which will be taken to represent the gravitational potential and matter density, respectively. DPMs of this theory are picked out by the field equations 40

\footnotetext{
${ }^{37}$ For details regarding Leibnizian, Galilean, and Newtonian structures, see [18, ch. 2].

${ }^{38}$ The exact mathematical forms of these groups are not relevant for our purposes - see [48, §3.1] for details.

${ }^{39}$ A Galilean structure is traditionally considered to be the 'most appropriate' spacetime setting for NGT, for in this case structure symmetries and dynamical symmetries (are claimed to) coincide, thereby satisfying Earman's "adequacy conditions" on spacetime theories (see [18, §3.4]). For recent philosophical discussion calling into question whether this orthodoxy is correct, see [16, 31, 53, 58, 60, 66, 68]; I do not discuss further such matters in this paper.

${ }^{40}$ Here, $R_{b c d}^{a}$ is the Riemann tensor associated with the derivative operator $\nabla_{a}$ defined in the Galilean structure.
} 


$$
\begin{aligned}
R_{b c d}^{a} & =0, \\
h^{a b} \nabla_{a} \nabla_{b} \varphi & =4 \pi \rho .
\end{aligned}
$$

(17) imposes flatness of $\nabla_{a} ;(18)$ is the Newton-Poisson equation. Finally, the gravitational force on a point (test) particle of mass $m$ is given by $-m h^{a b} \nabla_{b} \varphi$; it follows from Newton's second law that, if this particle is subject to no forces except gravity, and given that it has four-velocity $\xi^{a}$, then it satisfies

$$
-\nabla^{a} \varphi=\xi^{b} \nabla_{b} \xi^{a}
$$

Note that all elements of the Galilean structure feature in these dynamical equations; one can use this structure to offer a qualified explanation (in the sense of $\$ 4$ of the form of these dynamical laws.

Newtonian mechanics set in Galilean spacetime is a case in which structure symmetries coincide with dynamical symmetries ${ }^{41}$ Now consider a more nuanced case, in which dynamical symmetries constitute a proper subset of structure symmetries. One illustration of this is Newtonian mechanics set in a Leibnizian structure. KPMs of this 'theory' are tuples $\left\langle M, t_{a b}, h^{a b}, \varphi, \rho\right\rangle$ with $\left\langle M, t_{a b}, h^{a b}\right\rangle$ a Leibnizian structure, and $\varphi$ and $\rho$ defined as in the Galilean case; DPMs are (allegedly) picked out by (17)-(19). For the sake of argument granting that such a 'theory' is coherent ${ }^{42}$ we have a case in which dynamical symmetries are a proper subset of structure symmetries. What I contend here is that, in spite of the fact that structure symmetries and dynamical symmetries do not coincide, the fact that the Leibnizian structure still features in the DPMs of this theory means that it can still offer a partial (but not complete, since the laws also advert to other structure) explanation of the dynamical behaviour of matter in this case, in the qualified sense delineated in $\$ 4$ above.

Next consider the case in which dynamical symmetries are a proper superset of structure symmetries ${ }^{43}$ An illustration of such a scenario is Newtonian mechanics set in a Newtonian structure. In this case, KPMs are tuples $\left\langle M, t_{a b}, h^{a b}, \nabla_{a}, \sigma^{a}, \varphi, \rho\right\rangle$, where $\left\langle M, t_{a b}, h^{a b}, \nabla_{a}, \sigma^{a}\right\rangle$

\footnotetext{
${ }^{41}$ Setting aside the issues indicated in footnote 39

${ }^{42}$ Indeed, I here include scare quotes on the word 'theory', as there are good grounds to question whether such a 'theory' is really coherent, since it does not have sufficient structure in its KPMs to be able to write down the dynamical equations used to fix its DPMs - cf. [55, p. 6]. (Belot puts the point pithily, when he accuses those working with such theories of "arrant knavery" [5, p. 571]; for further related discussion, cf. [16, pp. 268269].)

${ }^{43}$ On this possibility, cf. Pooley's discussion at [48, p. 94].
} 
is a Newtonian structure (in which the integral curves of $\sigma^{a}$ are taken to represent the worldlines of the persisting points of Newtonian absolute space), and $\varphi$ and $\rho$ are understood as above; DPMs of this theory are again picked out by $(17)-(19)$. Though this theory is coherent ${ }^{44}$ as Earman states [18, $\left.\S 3.4\right]$, there is a sense in which it is nevertheless malformed, for the dynamical laws do not advert to all the Newtonian structure available in the KPMs of the theory (it is this which results in dynamical symmetries being a proper superset of structure symmetries). While I concur with Earman on this point, what I wish to register here is that, in this case, Newtonian structure may still be appealed to in explanations of the form of the dynamical laws governing matter fields - it is just that this structure has other, redundant explanatory apparata available to it (viz., the $\sigma^{a}$ field).

Thus, on QGA, in the case in which dynamical symmetries are a subset of structure symmetries, the relevant structure (whether metric, or e.g. Leibnizian/Galilean/Newtonian) may feature in partial explanations of the dynamical behaviour of matter. In the case in which dynamical symmetries are a superset of structure symmetries, by contrast, the relevant structure may feature in total but redundant explanations of the dynamical behaviour of matter. Note that Jacobson-Mattingly theory of 5.1 .2 instantiates the former case, in which dynamical symmetries are a subset of metric symmetries. ${ }^{45}$

Finally, consider the case in which dynamical symmetries partially overlap with structure symmetries - i.e., are neither a subset nor a superset of structure symmetries. One example of this is LAS, presented in 5.1.1. In this case, dynamical symmetries include the Euclidean transformations; symmetries of the Minkowski metric field $\eta_{a b}$ are the Poincaré transformations. The intersection of the Euclidean and Poincaré groups is the group of translations and spatial rotations (cf. [52, $\S \mathrm{B}]$ ); therefore, the corresponding degrees of freedom associated to the $\eta_{a b}$ field may still be used to account for these dynamical symmetries, in this case. (Though of course, an obvious question arises: why not instead appeal to $\delta_{a b}$ when giving this kind of qualified explanation of dynamical symmetries in this case?)

\footnotetext{
${ }^{44}$ At least on QGA - it is questionable whether this theory is coherent on the dynamical approach, according to which (as discussed above) metric/structure symmetries in theories with fixed metric/structure (such as both SR and NGT) just are dynamical symmetries. Cf. [10, §3.1].

${ }^{45}$ Though in this case the theory is coherent, in a way that arguably NGT set in a Leibnizian structure is not - cf. footnote 42 .
} 


\subsubsection{Confident and cautious qualified approaches}

Suppose that one embraces QGA, and suppose that one is considering a theory in which the metric/structure under consideration can be appealed to in order to offer a qualified explanation of the symmetries of the dynamical laws governing matter fields. For example, suppose that one is considering theories such as KGS, or Newtonian mechanics set in a Galilean structure. Even in such cases, there exists a further question relevant to the chronogeometric significance of this metric/structure, on which one might take different views. Namely: do there actually exist rods and clocks which survey this metric/structure?

Different possible answers to this question distinguish two subviews within QGA. On the one hand, one might maintain that, when the metric/structure features in a qualified explanation of the symmetries of the dynamical laws governing matter fields in the above sense, there always exist physical rods and clocks built from matter fields which survey that metric/structure. Call this view confident $\boldsymbol{Q} \boldsymbol{G A}$ On the other hand, one might reject the claim that, when the metric/structure features in a qualified explanation of the symmetries of the dynamical laws governing matter fields in the above sense, there always exist physical rods and clocks built from matter fields which survey that metric/structure. Call this view cautious $\boldsymbol{Q} \boldsymbol{G A}$.

Clearly, in order to call into question confident QGA, it suffices to present a single problem case. In fact, there exist several such cases; here I mention two. First, Pitts presents in [47] the example of universally coupled massive scalar gravity. In such theories, there exist two Lorentizan metic fields: a dynamical field $g_{a b}$, and a fixed Minkowski metric field $\eta_{a b}$; the Lagrangian includes the following graviton mass piece 47

$$
\mathcal{L}_{\text {mass }}=\frac{m^{2}}{64 \pi G}\left[\frac{\sqrt{-g}}{w-1}+\frac{\sqrt{-g}^{w} \sqrt{-\eta}^{1-w}}{w(1-w)}-\frac{\sqrt{-\eta}}{w}\right]
$$

(Here, $w$ is a free parameter, which may be fixed to yield specific theories.) The important point to note about such theories is put clearly by Pitts: "Massive scalar gravity lacks Minkowskian behav-

\footnotetext{
${ }^{46}$ Arguably, Maudlin falls into this camp, for he both (a) speaks of restricting dynamical equations in SR to those which couple universally to $\eta_{a b}$, thereby placing him in QGA (cf. footnote 34); and (b) argues that, in any model of SR, there exists a clock which satisfies the clock hypothesis, and thereby (by definition) correctly reads off intervals along its worldline as given by the metric field (cf. [38, ch. 5]). There are good reasons to doubt (b) - cf. [39, discussed further below.

${ }^{47}$ For the full details, see [4].
} 
ior of rods and clocks, though it has the Minkowski metric (among other things) and the Poincaré symmetry group. ... [T] he chronogeometrically observable conformally flat metric $g_{a b}=\hat{\eta}_{a b}(-g)^{1 / 4}$ isn't clearly the One True Geometry." [48 [47, p. 6] Thus, theories of this kind appear to pose problems for confident QGA, for rods and clocks generally do not survey $\eta_{a b}$, in spite of the fact that this field couples to the matter fields in the theory, and so may feature in a qualified explanation of their symmetries.

As a second example, the authors of [39] demonstrate, drawing upon recent work by Asenjo and Hojman [3], that there should be no expectation that physical rods and clocks (such as light clocks) correctly survey the metric field $g_{a b}$ of GR in particular solutions of this theory - namely in rotating solutions, such as the Gödel and Kerr solutions. The reasons are subtle, but essentially involve the fact that physical propagating media, such as light waves, do not travel at a fixed speed in such solutions, but rather manifest spacetime locationdependent propagation speeds. The central point here is a simple one: there is again reason to doubt confident QGA, for in these cases one has dynamical equations governing matter fields which feature coupling to $g_{a b}$, so that this metric field may feature in a qualified explanation of the symmetries of these equations and the behaviour of matter fields; nevertheless, rods and clocks do not survey this metric field, so that the chronogeometric significance of this field is questionable.

For these reasons, I take it that cautious QGA is to be preferredno a priori assumptions should be made regarding the behaviour of physical rods and clocks, even in cases in which a partial explanation of (e.g.) the symmetries of the dynamical equations in the theory under consideration via a given metric/structure is possible. In the remainder of this paper, I set this distinction aside for simplicitythough (for the above reasons) it should be taken that reference to QGA always means reference to cautious QGA.

\subsection{Two miracles, reprise}

With these subtleties regarding QGA addressed, I close this section by arguing that this approach does not account for MR1 and MR2; indeed, there is a sense in which MR1 and MR2 are more miraculous on QGA, than on the dynamical approach.

To see this, consider first SR on QGA. As in the case of the dynamical approach, on QGA it is conspiratorial - a 'miracle'- that

\footnotetext{
${ }^{48}$ Indices in this passage have been altered for consistency with the present paper; there is no change in content.
} 
all dynamical laws manifest the same symmetry properties, for recall that, unlike UGA, QGA seeks no explanation for this coincidence from within SR, in terms of $\eta_{a b}$. Put in other words, it is a brute fact on QGA that we do not consider other structures, such as $\delta_{a b}$, to which the matter fields in the theory could couple, and as a result of which coupling their dynamical laws would manifest different symmetries. Thus, MR1 holds also on QGA.

Since the advocate of QGA also considers even fixed metric structure such as $\eta_{a b}$ to be ontologically autonomous, however, a second coincidence arises even in SR: why is it that the symmetries of this metric field coincide with the symmetries of all dynamical laws? Clearly, this is just MR2 - again, another way to put the question is the following: why should the structure to which all dynamical laws for matter fields 'advert' be precisely the designated metric structure under consideration? From this, we see therefore that on QGA, both MR1 and MR2 hold even in the SR context. Since the dynamical approach faces the single miracle MR1 in SR (since it ontologically reduces metric structure in this theory to dynamical symmetries), this is, arguably, reason to favour the dynamical approach over QGA in SR.

In the GR context, QGA also faces both MR1 and MR2 - for exactly the reasons delineated in $\$ 3.2 .2$. Given this, a new question arises: given that both the dynamical approach and QGA agree in the GR context that the $g_{a b}$ field cannot be ontologically reduced to matter fields, and that both MR1 and MR2 hold in that context, is there really such a difference between the views, in this case? Absent the story of ontological reduction, there appears to be very little between the views. In light of this, I make the following claim: While the dynamical approach and $\boldsymbol{Q} \boldsymbol{G A}$ are distinct in the context of theories with fixed metric structure such as $S R$ (for they make different ontological claims regarding this fixed structure), they are not distinct in the context of theories with dynamical metric structure, such as GR 49

\section{The geodesic principle}

So far, I have: (a) clarified the distinction between the dynamical and geometrical approaches - the latter itself coming in two distinct

\footnotetext{
${ }^{49}$ Again, I am grateful to Oliver Pooley for impressing this point upon me. In this regard, cf. [48, p. 63], where Pooley writes, "What, then, is at stake between the metricreifying relationalist and the traditional substantivalist? Both parties accept the existence of a substantival entity, whose structural properties are characterised mathematically by a pseudo-Riemannian metric field and whose connection to the behaviour of material rods and clocks depends on, inter alia, the truth of the strong equivalence principle. It is hard to resist the suspicion that this corner of the debate is becoming merely terminological."
} 
varieties: UGA and QGA; (b) argued that while QGA is viable, UGA is not; (c) demonstrated that MR1 and MR2 hold both on the dynamical approach and on QGA; (d) argued that there is no difference between QGA and the dynamical approach in the context of GR. In this section, I consider the connections between this work, and recent and important results on the geodesic principle. I also reflect upon work by Knox [32, 30, 31] and Weatherall [69, §6] pertinent to the themes of this paper.

I begin with the geodesic principle. Contemporary work on this result stems largely from a 1975 theorem of Geroch and Jang [23]. Though more sophisticated extensions of this result now exist (in particular, see [19, 24]), I focus for the time being upon the Geroch-Jang theorem itself; this reads as follows ${ }^{50}$

Theorem 1 (Geroch and Jang (1975)) For a given $\left\langle M, g_{a b}\right\rangle$, where $g_{a b}$ is a Lorentzian metric field on $M$, let $\gamma: I \rightarrow M$ be a smooth, embedded curve. Suppose that, given any open subset $O$ of $M$ containing $\gamma[I]$, there exists a smooth, symmetric field $T^{a b}$ with the following properties:

1. $T^{a b}$ satisfies the strengthened dominant energy condition, i.e. given any timelike vector $\xi^{a}$ at any point $p \in M, T^{a b} \xi_{a} \xi_{b} \geq 0$ and either $T^{a b}=0$ or $T^{a b} \xi_{b}$ is timelike;

2. $T^{a b}$ satisfies the conservation condition, i.e. $\nabla_{a} T^{a b}=0$;

3. $\operatorname{supp}\left(T^{a b}\right) \subset O$; and

4. there is at least one point $p \in O$ for which $T^{a b}(p) \neq 0$.

Then $\gamma$ is a timelike curve that may be reparameterised as a geodesic.

The Geroch-Jang theorem makes precise the essence of the geodesic principle: that small bodies move on geodesics. In [69, §6], Weatherall draws a number of philosophical lessons regarding geodesic theorems such as the above (and its more sophisticated successors), which he takes to be consonant with the dynamical approach; it is to these putative lessons that I now turn 51 Begin with Weatherall's summary

\footnotetext{
${ }^{50}$ Here, I use the notation of [69, p. 6].

${ }^{51}$ For Brown's own discussion of the geodesic principle, see [6, §9.3]. With Brown's central contention - that geodesic motion of small bodies in GR is a consequence of the Einstein field equations, and is therefore automatic in GR, in a way that it is not in antecedent theories ("It is no longer a miracle." [6, p. 163])—Weatherall is in disagreement, for (a) geodesic motion is, in fact, independent of the Einstein field equations; (b) similar results can be derived in other theories, e.g. NGT, and Newton-Cartan theory. (For the details of Newton-Cartan theory, in which the gravitational potential $\varphi$ of NCT is absorbed
} 
of the import of results such as the Geroch-Jang theorem 52

[E]stablishing that small bodies respect the inertial structure encoded by a given derivative operator $\nabla_{a}$ requires one to establish that the $T^{a b}$ field associated with matter is divergence-free, or "conserved", with respect to $\nabla_{a}{ }_{53}^{53}[69$, p. 36]

Weatherall takes the fact that $T^{a b}$ is conserved with respect to a specific derivative operator $\nabla_{a}$ to deliver a connection between satisfaction of the geodesic principle and spacetime geometry - with this being particularly apparent if that derivative operator $\nabla_{a}$ is that which is compatible with some metric field: [69, p. 38]

From this perspective it is also fair to say that, as Brown argues in Physical Relativity, spacetime structures such as the metric may be viewed as "a codification of certain key aspects of the behaviour of particles and fields" (p. 142), at least as regards the link between free, small-body motion and the privileged class of curves picked out by a metric and/or derivative operator.

Though I am in agreement with Weatherall as far as the above statements go, there remains more to be said here, on two fronts. First, though it is true that some connection between matter fields and geometry is forged insofar as the stress-energy tensor associated with these fields is conserved with respect to a specific derivative operator, and moreover insofar as that matter thereby follows geodesics of that derivative operator, in accordance with the Geroch-Jang theorem (or its extensions), thus far the connection proceeds in terms of the motion of small bodies alone. To move from such results regarding the geodesic motion of small bodies, to the behaviour of matter fields tout court, is in effect to demand that the local symmetry properties of all matter fields be derivable from such geodesic motions; that is, it is, in effect,

into a (curved) derivative operator, see [37, ch. 4].) For Weatherall's work on the geodesic principle, see 69, 63, 62, 64, 70; I am in agreement with him on these matters. Also worthy of mention in this regard are remarks in a similar vein to (a) made by Pooley [48, p. 543]; and an earlier paper of Malament [36, in which it is pointed out (pace Brown) that geodesic motion in GR follows only on the assumption of the strengthened dominant energy condition.

${ }^{52}$ Here, Weatherall's notation has been amended slightly: I use ' $\nabla a$ ' rather than ' $\nabla$ '.

${ }^{53}$ In addition to the satisfaction of the strengthened dominant energy condition - again, see the Geroch-Jang theorem as stated above. 
to demand a proof of a result akin to Schiff's conjecture ${ }^{54}$ Only in that case could something like the SEP be delivered by this work on the geodesic principle 55

Second, it is important to be clear that this work does not provide a resolution to MR1. Even supposing that a connection is forged between geodesic motion and the local behaviour of matter fields more generally (à la Schiff's conjecture), that the mystery of MR1 remains can be demonstrated through asking the following question: why should all matter fields have associated stress-energy tensors, the divergences of which vanish with respect to the same derivative operator? If this were not the case, then it need not be the case that all matter fields survey the same 'practical geometry', in the manner explicated by Weatherall. Though it is true that, as Weatherall observes [69, p. 11], the Einstein field equations tell us (via the contracted Bianchi identity) that the covariant divergence of the total stress-energy content of any particular solution of GR vanishes, this is (again, as Weatherall observes - see [69, p. 12]) insufficient to infer that the divergences of the stress-energy tensors associated with all individual matter fields vanish with respect to the same derivative operator. Thus, these results on the geodesic theorem do not place

\footnotetext{
${ }^{54}$ In the words of Thorne et al., "Schiff's conjecture states that any complete and selfconsistent gravitation theory that obeys [the weak equivalence principle] must also, unavoidably, obey [the strong equivalence principle]" (emphasis in original) [59, p. 3575]. In turn, the weak equivalence principle is defined as follows: "If an uncharged test body is placed at an initial event in spacetime, and is given an initial velocity there, then its subsequent worldline will be independent of its internal structure and composition" (emphasis in original) [59, p. 3571]; the strong equivalence principle is defined as: "(i) [The weak equivalence principle] is valid, and (ii) the outcome of any local test experiment - gravitational or nongravitational - is independent of where and when in the universe it is performed, and independent of the velocity of the (freely falling) apparatus" [59, p. 3572]. For the original presentation of Schiff's conjecture, see [54, p. 343]; for ensuing discussion and attempted proofs of restricted versions of the conjecture, see [13, 35, 43, 59. Clearly, the version of Schiff's conjecture under consideration in this paper is different to that above - the gap to be bridged here is between the geodesic motions of small bodies, and the symmetries of matter fields tout court.

${ }^{55}$ Geroch and Weatherall demonstrate in [24] that source-free Maxwell fields 'track' null geodesics - a new result. Since the geodesic theorems demonstrate that massive matter moves on timelike geodesics, this gives access to both conformal and projective structure, respectively. One might think, therefore, that one may appeal to the Ehlers-Pirani-Schild result [20] (itself a generalisation of Weyl's theorem - cf. [72]), that (subject to extra constraints) conformal and projective structure fixes metric structure, to strengthen the connection between these geodesic theorems and geometry. While such results do indeed yield a further sense in which local geometry may be inferred from geodesic motions, they continue to leave unbridged the gap between the geodesic motions of small bodies, and the local dynamics of matter tout court. That is, Schiff's conjecture remains unproven, in general.
} 
sufficient restrictions on the behaviour of even small bodies built from different matter fields in order to resolve MR1.

The situation regarding the bearing of these results upon MR2 is more nuanced. Suppose that if the dynamical laws governing matter fields all manifest the same symmetries, then the stress-energy tensors associated with such matter fields (which satisfy the strengthened dominant energy condition, and the other conditions of the GerochJang theorem and its generalisations) have covariant divergences which vanish with respect to the same derivative operator. Now suppose that the dynamical laws governing matter fields all manifest the same symmetries. Then (by the above), the stress-energy tensors associated with such matter fields have covariant divergences which vanish with respect to the same derivative operator. Then, divergence of the total stress energy tensor (being a sum of the stress-energy tensors associated with the individual matter fields) with respect to this same derivative operator will also vanish; so, via the Einstein field equations, the left-hand side of the field equations will also have vanishing divergence with respect to this derivative operator-implying that the derivative operator is compatible with the metric field appearing in the Einstein tensor. In that case, small bodies built from all matter fields 'track' geodesics of a derivative operator associated with the Lorentzian metric field appearing in the Einstein field equations. In turn, one expects that in such a case the symmetries of the dynamical laws governing matter fields, and of this metric field, coincide, thereby delivering MR2. Of course, this reasoning is heuristic - but renders it prima facie plausible that these results regarding the geodesic principle may have application in resolving MR2.

In any case, let us now set aside these considerations regarding MR1 and MR2, and focus upon Weatherall's general morals drawn in [69, §6]. Consider the following passage:

$[T]$ he reason that a metric (or metrics) and derivative operator are able to codify the behavior of (generic) matter in the way characterized by the geodesic principle is precisely that that metric and derivative operator are the ones that appear in the dynamics of (all) matter in the relevant ways. And this, I think, is ultimately what is at the heart of the matter.

As I see it, the most perspicuous explication of what one means, or at least what one should mean, by the claim that spacetime has some geometry, represented by a given metric (or metrics) and derivative operator, is precisely that one can express the dynamics of (all) matter in such a way that all inner products are taken relative to that metric 
and all derivatives are taken relative to that derivative operator. This is the physical content of the claim that there are facts about distances, angles, and duration: physical processes occur in such a way that changes in a quantity at a time depend on the state of that quantity and those facts about distances, angles, and duration. And so, one is left with the conclusion that spacetime structures codify certain facts about the behavior of matter because the dynamics of (all) matter is adapted to those spacetime structures, which is just another way of saying that spacetime has that geometry. [69, pp. 39-40]

Though I am essentially in agreement with Weatherall on these matters, three points are important to note regarding this passage. First, and most straightforwardly, Weatherall (correctly) makes no appeal to UGA - he makes no claim to the effect that the metric field constrains all possible dynamical equations in a given theory, such that assumptions about the symmetry properties of those laws need not be made.

Second, nothing in this passage commits Weatherall either to the dynamical approach, or to QGA. Insofar as Weatherall takes e.g. NGT set in a Newtonian structure to be a coherent theory, there is perhaps some reason to take him to favour the latter, for recall that the coherence of this theory is questionable on the dynamical approach - cf. [10, $\S 3.1]_{56}^{56}$ Even in this case, however, one might take Weatherall's anticipated assessment that this theory is 'theoretically equivalent' (in a technical, category-theoretic sense -cf. [65, 67, 71]) to NGT set in a Galilean structure, combined with an implicit commitment to such theoretical equivalence being sufficient for physical equivalence, to indicate that he does not consider such to be the case-meaning that perhaps he should be regarded as siding with advocates of the dynamical approach after all 57

\footnotetext{
${ }^{56}$ In more detail, recall from footnote 44 that, on the dynamical approach, metric/structure symmetries in theories with fixed metric/structure just are dynamical symmetries - so how could it be the case that there exists a theory in which such symmetries do not coincide?

${ }^{57} \mathrm{I}$ concede that it is somewhat strained to seek to read Weatherall as an advocate of the dynamical approach; a reading on which he endorses something like QGA is more natural. Nevertheless, it is at least worth noting that advocation of the dynamical approach is consistent with Weatherall's writings. (Moreover - and interestingly-Weatherall has questioned in personal communication whether fixed metric structure, such as the Minkowski metric field of SR, should be regarded as being ontologically autonomous - in which case, his views are arguably closer to the dynamical approach than one might initially think. Whether, however, it is best to read Weatherall as endorsing the dynamical approach
} 
Third, Weatherall's views as expressed in the above passage are very consonant with the 'spacetime functionalism' of Knox [32, 30, 31], according to which "the spacetime role is played by whatever defines a structure of local inertial frames" [32, p. 22] (cf. \$1). To see this, some details regarding this programme of Knox must be recalled. Note first that, in GR, the chronogeometricity of the metric field precisely guarantees that this field be considered spatiotemporal, in Knox's sense. The reason is that, locally, the symmetries of the dynamical metric field coincide with those of the dynamical equations governing matter fields; in any frame in which these dynamical equations take their simplest form, the metric field itself takes the form diag $(-1,1,1,1)$. Thus, the metric field picks out a structure of local inertial frames-if one characterises such frames as those in which dynamical equations for non-gravitational fields take their simplest form (cf. [30, §2]).

Now recall that, for Weatherall, "what one means, or at least what one should mean, by the claim that spacetime has some geometry, represented by a given metric (or metrics) and derivative operator, is precisely that one can express the dynamics of (all) matter in such a way that all inner products are taken relative to that metric and all derivatives are taken relative to that derivative operator" [66, p. 40]. But, so coupling the dynamical equations governing matter fields will in general ensure that those equations have certain local symmetry properties - as, for instance, our discussion of KGG illustrated. In particular, it will in general ensure that metric symmetries coincide (locally) with dynamical symmetries - that is, it will ensure that the metric field qualifies as spatiotemporal, on Knox's programme 58 Thus, when Weatherall states that such coupling is sufficient for "spacetime to have some geometry", I take it that he is endorsing a view very much akin to Knox's spacetime functionalism 59

versus e.g. the version of the geometrical approach due to Janssen [29, 4, 28, in which the ontological autonomy of the metric field in e.g. SR is denied, remains unclear absent further work. Since the issues here are subtle, and it would take significant work to do justice to Janssen, these matters will have to wait for a future piece.)

${ }^{58}$ This coupling will ensure that a necessary condition on the metric field's having chronogeometric significance is satisfied - cf. \$3.

${ }^{59}$ Of course, it is also worth remaining conscious of the differences between Knox and Weatherall-for example, Weatherall makes no explicit commitment to inertial structure as the sine qua non of spacetime. 


\section{Conclusions}

In the context of SR (and of theories with a fixed metric/structure 60 more generally), advocates of the dynamical approach maintain that such a metric/structure is ontologically reducible to (symmetries of the dynamical laws governing) non-gravitational fields. By contrast, in the context of GR (and of theories with a dynamical metric/structure more generally), no such claim is made on the part of advocates of the dynamical approach. As a result of this, the dynamical approach arguably collapses into QGA in the GR context. While the dynamical approach is distinct from UGA in both SR and GR, there are good reasons to doubt the plausibility of UGA.

On QGA, we can appeal to the metric field of e.g. SR or GR to explain certain universal facts about the dynamics of matter fieldsbut only once further restrictions on the allowed class of models under consideration are imposed (for example, assumptions regarding the symmetries of the dynamical laws for non-gravitational fields, orrelatedly-assumptions of the universal coupling of the metric field under consideration to the matter fields in those dynamical equations, etc.). Thus, on both the dynamical approach and QGA, as yet no complete account of MR1 and MR2 exists within GR. Indeed, while the dynamical approach faces only MR1 in the context of SR, QGA faces both MR1 and MR2 in that theory; arguably, this reduction in the number of 'conspiracies' in SR constitutes reason to favour the former view over the latter. While work on geodesic principles establishes some connection between the dynamics of matter and the metric field of GR, this is in itself insufficient to account for MR1. Though there exist some hints that such results may be used to resolve MR2, more remains to be done in rendering these connections precise.

Weatherall may be understood as embracing Knox's spacetime functionalism, alongside either the dynamical approach, or QGA. Since both the dynamical approach and QGA are defensible, this is unproblematic. Indeed, arguably the geometrical approach has been written off too quickly by advocates of the dynamical view, as a result of a lack of appreciation of the viability of QGA. While I incline to the dynamical view - essentially on grounds of ontological parsimony in theories such as SR - I hope this paper may be of some value in demonstrating that the views of essentially all parties in this debate do not stand in such a state of conflict as one may prima facie be inclined to think.

\footnotetext{
60 'Structure' construed here in the sense of $\$ 5.2 .2$
} 


\section{Acknowledgments}

I am grateful to Erik Curiel, Patrick Dürr, Niels Linnemann, Tushar Menon, Oliver Pooley, and Jim Weatherall for invaluable comments on earlier drafts of this paper; to Harvey Brown, Jeremy Butterfield, Dennis Lehmkuhl, and Brian Pitts for fruitful discussions on the dynamical approach; and to Jim Weatherall (again) for asking a pertinent question which motivated my writing this paper. I am also indebted to audiences in Bern, Bonn, Cambridge, Exeter, Kraków, and London for useful feedback. I am supported by an AHRC grant at the University of Oxford; a senior scholarship at Hertford College, Oxford; and a fellowship with the Space and Time After Quantum Gravity project at the University at Illinois at Chicago - the latter funded by the John Templeton Foundation.

\section{References}

[1] Pablo Acuña, "Minkowski Spacetime and Lorentz Invariance: The Cart and the Horse or Two Sides of a Single Coin?", Studies in History and Philosophy of Modern Physics 55, pp. 1-12, 2016.

[2] J. L. Anderson, Principles of Relativity Physics, New York: Academic Press, 1967.

[3] Felipe A. Asenjo and Sergio A. Hojman, "Do Electromagnetic Waves Always Propagate Along Null Geodesics?", Classical and Quantum Gravity 34, 205011, 2017.

[4] Yuri Balashov and Michel Janssen, "Presentism and Relativity", British Journal for the Philosophy of Science 54(2), pp. 327-346, 2003.

[5] Gordon Belot, "Geometry and Motion", British Journal for the Philosophy of Science 51, pp. 561-595, 2000.

[6] Harvey R. Brown, Physical Relativity: Spacetime Structure from a Dynamical Perspective, Oxford: Oxford University Press, 2005.

[7] Harvey R. Brown and Oliver Pooley, "The Origins of the Spacetime Metric: Bell's Lorentzian Pedagogy and its Significance in General Relativity", in Craig Callender and Nick Huggett (eds.), Physics Meets Philosophy at the Plank Scale, Cambridge: Cambridge University Press, 2001.

[8] Harvey R. Brown and Oliver Pooley, "Minkowski Space-Time: A Glorious Non-Entity", in Dennis Dieks (ed.), The Ontology of Spacetime, Elsevier, 2006. 
[9] Harvey R. Brown and James Read, "Clarifying Possible Misconceptions in the Foundations of General Relativity", American Journal of Physics 84(5), pp. 327-334, 2016.

[10] Harvey R. Brown and James Read, "The Dynamical Approach to Spacetime", in E. Knox and A. Wilson (eds.), The Routledge Companion to Philosophy of Physics, Oxford: Routledge, 2018. (Forthcoming.)

[11] Jeremy Butterfield, "Reconsidering Relativistic Causality", International Studies in the Philosophy of Science 21(3), pp. 295-328, 2007.

[12] Sean Carroll and Eugene Lim, "Lorentz-Violating Vector Fields Slow the Universe Down", Physical Review D 70, 123525, 2004.

[13] A. Coley, "Schiff's Conjecture on Gravitation, Physical Review Letters 49(12), pp. 853-855, 1982.

[14] Erik Curiel, "A Primer on Energy Conditions", in D. Lehmkuhl, G. Schiemann and E. Scholz (eds.), Towards a Theory of Spacetime Theories, Birkhäuser, pp. 43-104, 2017.

[15] Erik Curiel, "On Geometric Objects, the Non-Existence of a Gravitational Stress-Energy Tensor, and the Uniqueness of the Einstein Field Equation", Studies in History and Philosophy of Modern Physics, 2017. (Forthcoming.)

[16] Neil Dewar, "Maxwell Gravitation", Philosophy of Science 85, pp. 249-270, 2018.

[17] Neil Dewar and James Owen Weatherall, "On Gravitational Energy in Newtonian Theories", Foundations of Physics 48(5), pp. 558-578, 2018.

[18] John Earman, World Enough and Space-Time: Absolute Versus Relational Theories of Space and Time, Cambridge, MA: MIT Press, 1989.

[19] Jürgen Ehlers and Robert Geroch, "Equation of Motion of Small Bodies in Relativity", Annals of Physics 309, pp. 232-236, 2004.

[20] Jürgen Ehlers, Felix A. E. Pirani and Alfred Schild, "The Geometry of Free Fall and Light Propagation", in L. O'Reifeartaigh (ed.), General Relativity: Papers in Honour of J. L. Synge, Oxford: Clarendon Press, pp. 63-84, 1972.

[21] Albert Einstein, "What is the Theory of Relativity?", London Times, 1919.

[22] Michael Friedman, Foundations of Space-Time Theories, Princeton: Princeton University Press, 1983. 
[23] Robert Geroch and Pong Soo Jang, "Motion of a Body in General Relativity", Journal of Mathematical Physics 16(1), pp. 65-67, 1975 .

[24] Robert Geroch and James Owen Weatherall, "The Motion of Small Bodies in Space-Time", Communications in Mathematical Physics 364, pp. 607-634, 2018.

[25] Carl Hoefer, "Energy Conservation in GTR", Studies in History and Philosophy of Modern Physics 31, pp. 187-199, 2000.

[26] Nick Huggett, "Essay Review: Physical Relativity and Understanding Space-Time", Philosophy of Science 76, pp. 404-422, 2009.

[27] Ted Jacobson and David Mattingly, "Gravity with a Dynamical Preferred Frame", Physical Review D 64, 024028, 2001.

[28] Michel Janssen, "COI Stories: Explanation and Evidence in the History of Science", Perspectives on Science 10, pp. 457-522, 2002.

[29] Michel Janssen, "Drawing the Line Between Kinematics and Dynamics in Special Relativity", Studies in History and Philosophy of Modern Physics 40, pp. 26-52, 2009.

[30] Eleanor Knox, "Effective Spacetime Geometry", Studies in History and Philosophy of Modern Physics 44, pp. 346-356, 2013.

[31] Eleanor Knox, "Newtonian Spacetime Structure in Light of the Equivalence Principle", British Journal for the Philosophy of Science 65(4), pp. 863-880, 2014.

[32] Eleanor Knox, "Physical Relativity from a Functionalist Perspective", Studies in History and Philosophy of Modern Physics, 2017. (Forthcoming.)

[33] Vincent Lam, "Gravitational and Nongravitational Energy: The Need for Background Structures", Philosophy of Science 78, pp. 1012-1024, 2011.

[34] Dennis Lehmkuhl, "Mass-Energy-Momentum: Only there Because of Spacetime?", British Journal for the Philosophy of Science 62, pp. 453-488, 2011.

[35] Alan P. Lightman and David L. Lee, "Restricted Proof that the Weak Equivalence Principle Implies the Einstein Equivalence Principle, Physical Review D 8(2), pp. 364-376, 1973.

[36] David Malament, "A Remark About the "Geodesic Principle" in General Relativity", in M. Frappier, D. H. Brown and R. DiSalle (eds.), Analysis and Interpretation in the Exact Sciences: Essays 
in Honour of William Demopoulos, New York: Springer, pp. 245$252,2012$.

[37] David Malament, Topics in the Foundations of General Relativity and Newtonian Gravitation Theory, Chicago: Chicago University Press, 2012.

[38] Tim Maudlin, Philosophy of Physics Volume I: Space and Time, Princeton: Princeton University Press, 2012.

[39] Tushar Menon, Niels Linnemann and James Read, "Clocks and Chronogeometry: Rotating Spacetimes and the Relativistic Null Hypothesis", British Journal for the Philosophy of Science, 2018. (Forthcoming.)

[40] Hermann Minkowski, "Raum und Zeit", Physikalische Zeitschrift 10, pp. 104-111, 1909.

[41] Charles Misner, Kip Thorne, and John Wheeler, Gravitation, San Francisco: Freeman \& Co., 1973.

[42] Wayne C. Myrvold, "How Could Relativity be Anything Other Than Physical?", Studies in History and Philosophy of Modern Physics, 2017. (Forthcoming.)

[43] Wei-Tou Ni, "Equivalence Principles and Electromagnetism, Physical Review Letters 38(7), pp. 301-304, 1977.

[44] John D. Norton, "Why Constructive Relativity Fails", British Journal for the Philosophy of Science 59, pp. 821-834, 2008.

[45] J. Brian Pitts, "Absolute Objects and Counterexamples: JonesGeroch Dust, Torretti Constant Curvature, Tetrad-Spinor, and Scalar Density", Studies in History and Philosophy of Modern Physics 37(2), pp. 347-371, 2006.

[46] J. Brian Pitts, "Gauge-Invariant Localization of Infinitely Many Gravitational Energies from All Possible Auxiliary Structures", General Relativity and Gravitation 42, 2010.

[47] J. Brian Pitts, "Space-time Constructivism vs. Modal Provincialism: Or, How Special Relativistic Theories Needn't Show Minkowski Chronogeometry", Studies in History and Philosophy of Modern Physics, 2017. (Forthcoming.)

[48] Oliver Pooley, "Substantivalist and Relationist Approaches to Spacetime", in R. Batterman (ed.), The Oxford Handbook of Philosophy of Physics, Oxford University Press, 2013.

[49] Oliver Pooley, "Background Independence, Diffeomorphism Invariance, and the Meaning of Coordinates", in D. Lehmkuhl, G. Schiemann and E. Scholz (eds.), Towards a Theory of Spacetime Theories, Birkhäuser, pp. 105-144, 2017. 
[50] James Read, "Functional Gravitational Energy", British Journal for the Philosophy of Science, 2018. (Forthcoming.)

[51] James Read, "On Miracles and Spacetime", Studies in History and Philosophy of Modern Physics, 2018. (Forthcoming.)

[52] James Read, Harvey R. Brown and Dennis Lehmkuhl, "Two Miracles of General Relativity", Studies in History and Philosophy of Modern Physics, 2018. (Forthcoming.)

[53] Simon Saunders, "Rethinking Newton's Principia", Philosophy of Science 80, pp. 22-48, 2013.

[54] L. I. Schiff, "On Experimental Tests of the General Theory of Relativity, American Journal of Physics 28, pp. 340-343, 1960.

[55] Howard Stein, "Some Philosophical Prehistory of General Relativity", in J. Earman, C. Glymour and J. Stachel (eds.), Foundations of Space-Time Theories, Minnesota Studies in the Philosophy of Science, volume 8, Minneapolis: University of Minnesota Press, pp. 3-49, 1977.

[56] Syman Stevens, "The Dynamical Approach as Practical Geometry", Philosophy of Science 82, pp. 1152-1162, 2015.

[57] Syman Stevens, "Regularity Relationalism and the Constructivist Project", British Journal for the Philosophy of Science, 2017. (Forthcoming.)

[58] Nicholas J. Teh, "Recovering Recovery: On the Relationship Between Gauge Symmetry and Trautman Recovery", Philosophy of Science 85, pp. 201-224, 2018.

[59] Kip S. Thorne, David L. Lee, and Alan P. Lightman, "Foundations For a Theory of Gravitation Theories", Physical Review D 7, pp. 3563-3578, 1973.

[60] David Wallace, "Fundamental and Emergent Geometry in Newtonian Physics", British Journal for the Philosophy of Science, 2017. (Forthcoming.)

[61] James Owen Weatherall, "On (Some) Explanations in Physics", Philosophy of Science 78(3), pp. 421-447, 2011.

[62] James Owen Weatherall, "On the Status of the Geodesic Principle in Newtonian and Relativistic Physics", Studies in the History and Philosophy of Modern Physics 42(4), pp. 276-281, 2011.

[63] James Owen Weatherall, "The Motion of a Body in Newtonian Theories", Journal of Mathematical Physics 52(3), 032502, 2011.

[64] James Owen Weatherall, "A Brief Remark on Energy Conditions and the Geroch-Jang Theorem", Foundations of Physics 42(2), pp. 209-214, 2012. 
[65] James Owen Weatherall, "Are Newtonian Gravitation and Geometrized Newtonian Gravitation Theoretically Equivalent?", Erkenntnis 81(5), pp. 1073-1091, 2016.

[66] James Owen Weatherall, "Maxwell-Huygens, Newton-Cartan, and Saunders-Knox Space-Times", Philosophy of Science 83, pp. 82-92, 2016.

[67] James Owen Weatherall, "Understanding Gauge", Philosophy of Science 85(5), pp. 1039-1049, 2016.

[68] James Owen Weatherall, "A Brief Comment on Maxwell(/Newton)[-Huygens] Spacetime", Studies in History and Philosophy of Modern Physics, 2017. (Forthcoming.)

[69] James Owen Weatherall, "Conservation, Inertia, and Spacetime Geometry", Studies in History and Philosophy of Modern Physics, 2017. (Forthcoming.)

[70] James Owen Weatherall, "Inertial Motion, Explanation, and the Foundations of Classical Spacetime Theories", in D. Lehmkuhl, G. Schiemann and E. Scholz (eds.), Towards a Theory of Spacetime Theories, Birkhäuser, pp. 13-42, 2017.

[71] James Owen Weatherall, "Categories and the Foundations of Classical Field Theories", in E. Landry (ed.), Categories for the Working Philosopher, Oxford: Oxford University Press. (Forthcoming.)

[72] Hermann Weyl, "Zur Infinitesimalgeometrie: Einordnung der Projektiven und der Konformen Auffasung", Nachrichten von der Gesellschaft der Wissenschaften zu Göttingen, MathematischPhysikalische Klasse, Göttingen, pp. 99-112, 1921.

[73] Hermann Weyl, Mathematische Analyse des Raumproblems, lecture 3, Berlin, 1923. 